\title{
Data Driven Forecast of Droplet Combustion
}

\author{
Xiuqi Xi ${ }^{\mathrm{a}}$, José L. Torero and Wolfram Jahn ${ }^{\mathrm{b}}$ \\ ${ }^{a}$ University College London, London WC1E 6BT, UK \\ ${ }^{b}$ Departamento de. Ingeniería Mecánica y Metalúrgica, Pontificia Universidad Católica de Chile, Chile
}

\begin{abstract}
The characteristics of a diffusion flame resulting from the gasification of a condensed fuel are predicted from the synthesis of simple models and data. Combustion of a droplet in microgravity is used as a canonical configuration to illustrate the methodology. The simplicity of the spherical configuration and the detail of the measurements make the available experimental data ideal for this study. The approach followed combines the classical analytical solution first proposed by Spalding to describe the condensed phase gasification with a numerical method that describes the gas phase. Available data on flame geometry and regression rates are used to initialize the model and produce adequate predictions of the time evolution of all relevant variables. The method was shown to make proper predictions under numerous configurations and with very small computational cost.
\end{abstract}

Keywords: data assimilation; droplet combustion; hybrid model;

Colloquium: Fire Research

Total length of paper: 5744

Main body: 4055 ; Equations: $(10 \times 3+2) \times 7.6 \approx 244$; Nomenclature: None; Reference: $(18+2) \times 2.3 \times 7.6$ $\approx 350$; Tables: None; Figures and Captions: $(49+50+55+54+55+54+54+7 \times 10) \times 2.2+124 \approx 1095$ 


\section{Introduction}

It is generally difficult to make blind predictions of fluid flows, because the nonlinear nature of the governing equations makes them very sensitive to input parameters and initial and boundary conditions. This was established and quite convincingly demonstrated by atmospheric scientists some 50 years ago [1]. To overcome this intrinsic limitation of restricted predictability, it was proposed by meteorologists to continuously re-initialize forecasts with updated atmospheric measurements [2]. This technique, known as data assimilation, powers all existing operational weather forecasting systems, preventing the propagation of model sensitivities and the associated growth of simulation errors that eventually lead to chaotic behavior.

The complex coupling of momentum and energy equations with combustion and radiation models make forecasts of flow fields in reactive flows especially challenging (see e.g. [3]). If the aim of the forecast is to predict the flame characteristics resulting from the burning of solid or liquid fuel, an additional aspect has to be taken into account; in this case the production rate of combustible volatiles is part of the output of the forecast, and heat transfer into the condensed fuel and its gasification (or thermal degradation--i.e. pyrolysis) need to be added. This further complicates the matter as a positive feedback loop emerges where the heat from the flame conditions controls the rate of production of combustible volatiles, and the rate of production, in turn, conditions the heat output of the flame (and the flow field, as in such cases the flow is often driven by buoyancy rather than momentum).

A numerical model that would simulate the coupled gas phase-solid phase combustion problem from first principles in scenarios with a relevant physical scale (e.g. flame spread over a piece of furniture) is beyond the current capabilities of even the most advanced computers [4]. Even if such a simulation were attempted, its horizon of predictability would be limited. It is then only natural to extend the concept of data-driven simulations to combustion and fire modelling, in order to relax some 
of the restrictive physical models and expand the horizon of predictability.

Gao et al. present a comprehensive summary of the application of data assimilation techniques to premixed laminar combustion [5]. Yu et al. use the $G$-equation as a reduced order model to simulate a premixed turbulent flame, and assimilate synthetic data in order to estimate model parameters [6]. Labahn et al. perform high fidelity LES of a partially premixed turbulent flame in conjunction with data assimilation based on ensemble Kalman filtering [7]. Jahn et al. argue that fire growth can be modelled in many scenarios using a simple expression that correlates two parameters (spread velocity and burning rate) to the rate of heat released, and present an inverse modelling approach to estimate the parameters [8-10]. The results show the potential to substitute the near-wall region by some simple analytical model that forms parts of the forward model. In some cases adequate predictions were achieved, but in all cases computing times disabled the possibility of forecasting using these models.

All these attempts towards the use of data to drive condensed phase fuel combustion have addressed complex configurations, and thus, it has been difficult to establish the main factors disabling adequate and efficient forecasts. In this study, the simplest possible configuration (the burning droplet) that still exhibits the main features of condensed fuel combustion is analyzed. The objective is to define the framework and to establish the main issues that need to be resolved. With a validated framework in place, this methodology of coupling the gas and condensed phase by assimilating data can then be extended to more complex geometries and further applied to predictions of fire development.

\section{Droplet Combustion Model}

A mathematical representation of a conglomerate of physical and chemical processes that predicts their evolution in time is called a forward model. Droplet combustion in the absence of buoyancy can be described as a one-dimensional (advection?)-diffusion problem with reaction. An adequate forward model that incorporates these physical procedures is required first. For the forward model, there are 
two approaches that can describe the droplet combustion system, the classical analytical solution or Computational Fluid Dynamics (CFD). Currently, neither the classical analytical model or a CFD model can fully deliver a precise forward model. The approach followed here couples the analytical model and CFD together with data to deliver a forward model that can predict the main variables of the problem.

\subsection{Classical analytical model}

The analytical solution for droplet combustion in microgravity was originally proposed by Spalding [11]. The main assumptions of the model are that in quiescent microgravity a droplet can be considered spherical, and the quasi-steady-state assumption. The classical theory relates the mass flux of the fuel at the surface to the Spalding transfer number,

$$
B=\left[\Delta H f Y_{O \infty}+c_{p, g}\left(T_{\infty}-T_{s}\right)\right] /\left(L+Q_{\text {loss }}\right)
$$

where $\Delta H$ is the heat of combustion, $f$ is the stoichiometric coefficient, $Y_{O_{\infty}}$ is the ambient oxygen concentration, $T_{\infty}$ is the ambient temperature, $T_{s}$ is the surface temperature of the droplet, $c_{p, g}$ is the heat capacity for the gas, $L$ is the heat of gasification and $Q_{\text {loss }}$ is a term that amalgamates all heat transfer processes not considered, primarily heat losses from the droplet surface.

Once the mass flux is obtained, the droplet diameter $R$ can be calculated by integrating the droplet diameter change in time $t$, leading to the $R$-square Law as

$$
R^{2}=R_{0}^{2}-K t
$$

where $R_{0}$ is the initial droplet radius, and $K$ is a function of the fuel mass flux $\dot{m}^{\prime \prime}$ as,

$$
K=\left(2 \dot{m}^{\prime \prime} R\right) / \rho_{l}
$$

where is $\rho_{l}$ the density of the condensed phase. The flame stand-of ratio (FSR) as the ratio between the flame radius $r_{f}$ and droplet radius $R$ can also be

$$
r_{f} / R=\ln (B+1) / \ln \left(f Y_{O \infty} / Y_{F R}+1\right)
$$


where $Y_{F R}$ are the mass fractions of oxygen and fuel, respectively.

Even though the analytical solution can provide explicit expressions for different physical variables, the model does not deliver enough information to use it as forward model. The most restrictive factor is the quasi-steady assumption for the gas phase, especially in the context of predictive simulations. If adequate predictions are required, this time evolution needs to be captured. Given that the gasification process includes the time evolution of the condensed phase, there is potential for data assimilation to be used with the condensed phase model.

\subsection{Numerical model}

Another approach to describe the droplet combustion is using numerical methods. To simulate the full process of droplet combustion, both the condensed phase and the gas phase must be simulated in parallel. For the interface between the condensed phase and the gas phase, additional models have to be added to calculate the mass and heat transfer through the interface [12].

The numerical model is suitable as a forward model because the physics are correctly reproduced and the output is detailed enough. By adjusting the corresponding parameters by comparison to data, an appropriate result can be attained (one that represents reality to an acceptable degree). With the adjusted parameters, a prediction of the future state of the system may be made. As there is no need for a quasi-steady simplification, the time dependency is present in both the condensed and gas phase.

The main problem with a fully resolved numerical implementation of droplet combustion is that it takes a significant amount of time to run. Data assimilation is an iterative process, where the forward model is run sequentially with different sets of parameters until the model output matches the observations. Thus, if the forward model consumes significant time its potential as a forward model vanishes. Solving the interface between the condensed and gas phases requires significant computational resources, because the evaporation process is challenging, and an additional modelling 
step that consumes significant resources needs to be implemented. In view of this, a fully resolved numerical approach would be equally inappropriate for data-driven forecasting of droplet combustion.

\subsection{Coupled model}

Given that the analytical solution lacks a transient gas phase and the numerical solution requires large computational resources to treat the condensed phase and the interface, it is logical to combine both models to obtain a forward model. It is thus proposed here to solve the gas phase (including the flaming region) numerically and substituting the condensed and its immediate surroundings with a region of fixed size, where the analytical model is applied. By substituting the condensed and surrounding regions with the classical analytical region, the analytical method can have a dynamic gas phase and the numerical method can eliminate the computationally intensive regions.

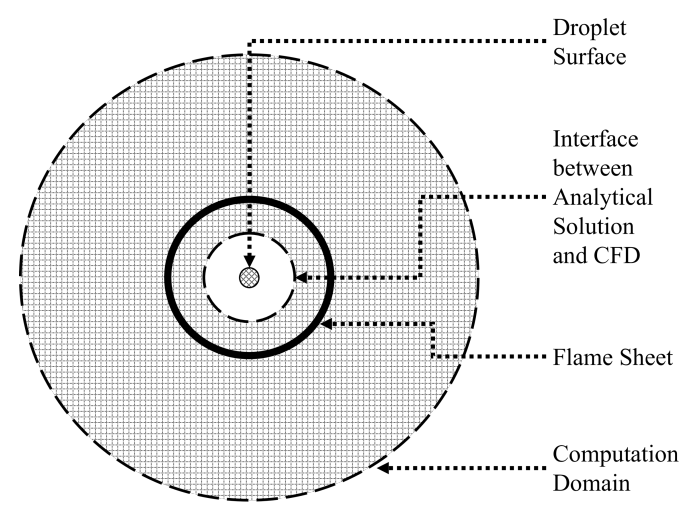

Figure 1: The schematic of the forward model coupled with the analytical model and the numerical model

Figure 1 shows a schematic of the proposed hybrid model. The location of the interface between the two regions is set to be the original radius of the droplet $R_{0}$. This way the location of the flame is inside the numerical region while the droplet is in the analytical region.

The interface represents the boundary condition for both the analytical region and numerical 
region. The boundary condition of each region is therefore controlled by the other region. The heat flux from the numerical region that will define the evaporation of fuel in the condensed (analytical) region is an output on each discretized boundary cell of the numerical solution. The mean of the maximum and minimum heat flux among all boundary elements at the interface between analytical and numerical regions during each time step is used as the input for analytical region. From the energy balance between the interface and droplet surface, the heat flux at the surface is given by

$$
\dot{q}^{\prime \prime}(R)=\dot{q}^{\prime \prime}\left(R_{0}\right) R_{0}^{2} / R^{2}
$$

From the analytical solution, the mass flux at the droplet surface is proportional to the heat flux at the surface according to the following expression obtained by means of a simple mass balance at the interface,

$$
\dot{m}^{\prime \prime}(R)=B \frac{\dot{q}^{\prime \prime}(R)}{Y_{O \infty} f \Delta H+c_{p g}\left(T_{\infty}-T_{b}\right)}
$$

With the mass flux at the interface, the boundary conditions (e.g. fuel fraction) for the numerical solution can be calculated.

$$
\begin{aligned}
Y_{F}= & \left(Y_{F R}+f Y_{O \infty}\right) \\
& \left\{1-\exp \left[-k_{g} \dot{m}^{\prime \prime} R^{2} /\left(c_{p g} R_{0}\right)\right]\right\}-f Y_{O \infty}
\end{aligned}
$$

Once the boundary conditions are determined, the numerical simulation can proceed and then again provide the heat flux towards the interface. A modified solver is developed based on the combustion solver that is incorporated in OpenFOAM.

\section{Parameters Determined with Observed Data}

There are many variables and uncertainties remaining in the coupled model. Still, it can be used to forecast the future state of the system because the data-driven process can "absorb" uncertainties and forecast the future states by correcting existing parameters using acquired data. However, the parameters that need to be determined are many, especially for the numerical region. Moreover, the 
result is generally sensitive to many parameters, and thus they need to be established with sufficient precision. An important aspect of data assimilation is that when data is used to correct parameters, if the forward model incorporates sufficient details of the physical and chemical processes, these parameters cannot be time dependent. Convergence towards a set of representative time-independent parameters is therefore necessary.

In principle, a single corrective parameter should suffice because if the evolution of the droplet diameter can be correctly predicted, then the boundary condition for the CFD solution should be the correct one, and thus the solution for the flame radius should be correct. This is not the case because the outer region, despite being simulated by CFD, includes many uncertainties and simplifications. The correction for the condensed phase is necessary to correct for all the uncertainties and simplifications in the inner domain. The CFD analysis in turn has simplified properties and chemistry, thus the second correction for the gas phase is necessary. This means that during the assimilation a minimum of two sets of data needs to be used: For the inner domain the coefficient $K$ (from the $R$ square law), and for the outer region the Spalding number $B$ are chosen as the data-driven parameters (denoted by $\hat{K}$ and $\hat{B}$, respectively). More parameters could be used if the two chosen parameters do not suffice. Various parameters can potentially be steered. In this work, examples are shown where these two parameters successfully steer the system in the right direction. A generalized model will be considered in future work.

For the inner region, the corrected parameter $\hat{K}$ is determined by using Eq. (1) and the observed droplet radius

$$
\hat{K}=\left(R_{\text {observed }}^{2}-R_{0}^{2}\right) / t
$$

For the outer region, the corrected parameter $\hat{B}$ is determined by measuring the flame radius. Both analytical and numerical solutions are viable for determining the flame location. Correspondingly, 
these two solutions can be used to obtain the corrected $B$ from the observed flame location. Analytically, the corrected $\hat{B}$ from Eq. (2) is

$$
\hat{B}=\exp \left[r_{f}^{\text {observed }} \ln \left(f Y_{O \infty} / Y_{F R}+1\right) / R\right]-1
$$

Numerically, the corrected $B$ is as follows

$$
\hat{B}=\mathbf{L}^{-1}\left(r_{f}^{\text {observed }}\right)
$$

where $\mathbf{L}$ corresponds to the CFD model that allows for estimating the flame location given a certain $B$ number. Eq. (10) thus represents the inverse process of estimating $B$ from observations of the flame location.

Due to the small number of parameters, a simple binary search algorithm that minimizes the Euclidean distance between predictions and observations is used to obtain an acceptable value for $\hat{B}$. The interval for the binary search algorithm is from 0 to the theoretical $B$ value from the analytical solution.

As the flame location can be predicted analytically or numerically, analytical and numerical methods can be used for the inverse and forward process. Thus, four combinations can be used to assimilate and predict the flame location. As shown in the discussion section, the combination of numerical assimilation and numerical predictions with numerical inverse model shows the best match to the experiments.

Finally, to enable the forward model to deliver a precise forecast, an adequate set of data is necessary.

\section{Data Assimilation Implementation with Experimental Observations}

The data used are from the Flame Extinguishment Experiment (FLEX), conducted on board the International Space Station by NASA [13-16]. The experiments used n-heptane, n-decane and n-octane droplets of initial size ranging from $0.85 \mathrm{~mm}$ to $4.36 \mathrm{~mm}$ and all the data provided are from free-floating 
combustion without any support (not attached to fibers or other filaments).

The measurements of the experiments included the multispectral and color cameras that record the droplet burning process. There are two direct measurements, the droplet diameter and flame location. The collected data is divided into two groups; the data used as inputs will be labeled the "observed data" and the data used as validation for the data-driven forecasts will be referred to as the "predicted data". Moreover, other outputs as species profiles are compared with a detailed simulation which will be explained in more detail in the next section.

For the assimilation, two droplet types are selected, n-heptane and n-decane. The original radius of the $\mathrm{n}$-heptane droplet is $1.74 \mathrm{~mm}$, and the original radius of the $\mathrm{n}$-decane droplet is $1.98 \mathrm{~mm}$.

\section{Results and Discussion}

The simulations performed for both droplets use the initial $5 \mathrm{~s}$ of the droplet size data to quantify $\hat{K}$, and the first observation of the flame location is used to obtain $\hat{B}$ to reduce the iteration times for the assimilation. Then, the forward model provides a prediction from 5 to $10 \mathrm{~s}$ with these values. The cutoff between the observation and prediction region is chosen as 5 s because by this time, the droplet has stopped expanding and shrinks steadily. In the observation period, the number of observations is enough to assimilate parameter and make proper predictions. A later cutoff can lead to a better prediction but the accuracy does not increase significantly and the prediction period (and thus the lead time) will be shortened. Figure 2 provides the droplet radius observations and predictions during the $10 \mathrm{~s}$ combustion period. 


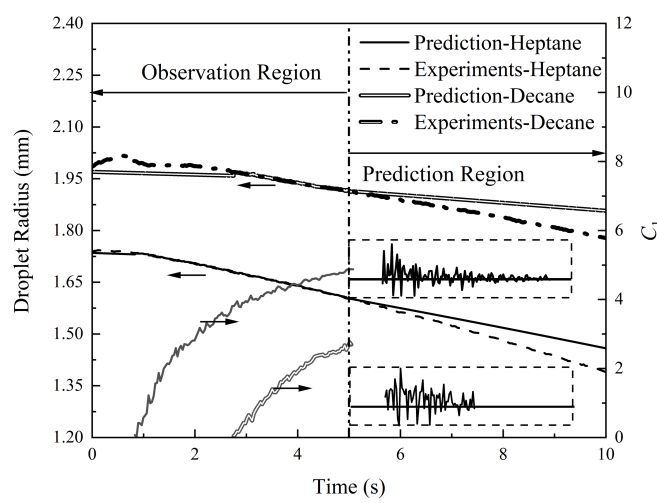

Figure 2: Droplet size observations and predictions for $10 \mathrm{~s}$ of $\mathrm{n}$-heptane and n-decane droplet combustion. The right axis shows the value of $C_{1}=\hat{K} / K$. Plots in the dashed boxes show the tendency of the displacement increment for each step. The upper box is for $n$-heptane and the lower is for $\mathrm{n}$-decane

As expected, Figure 2, shows that in the period of data assimilation (i.e. from 0 to $5 \mathrm{~s}$ ) there is an overlap between predictions and experimental data. This indicates that the assimilation is completed successfully. At the beginning of the combustion, the droplet will expand thermally and the $R$-square law cannot be applied. Thus, the valid observations start at $0.9 \mathrm{~s}$ for $\mathrm{n}$-heptane and for $\mathrm{n}$-decane starts at $2.8 \mathrm{~s}$. Then, from $5 \mathrm{~s}$ to $10 \mathrm{~s}$, there is a systemic drift between the experiments and forecast. The Root Mean Squared error (RMSE) normalized by the mean of the measurements between the prediction and observation is $2.2 \%$ for $n$-decane and $2.46 \%$ for $n$-heptane. Considering that both droplets share the same input parameters and that complex physics and chemistry plays a very significant role as the diameter of the droplet shrinks, these differences appear acceptable.

Once the corrected parameters are obtained, ratios between the calibrated and theoretical values ( $C_{1}=\hat{K} / K$ and $C_{2}=\hat{B} / B$ ) can be added to quantify the difference between the corrected result and the theoretical result. An important aspect of this process is the evaluation of the $C_{1}$ parameter as a function of time, leading to convergence if the assimilation process is correct. Figure 2 shows the value 
of $C_{1}$ on the right axis. As can be seen, the value of $C_{1}$ is fuel dependent, but nevertheless converges in both cases ( $C_{1} \approx 5$ for n-heptane and $C_{1} \approx 2.5$ for n-decane). The tendencies of the displacement increment for each step are shown in the dashed boxes. Both cases exhibit convergency and n-heptane case has longer assimilation period compared to n-decane case. The convergence of $C_{1}$ indicates that a time-independent parameter is successfully estimated.

Figure 3 shows the observations and predictions of the FSR. The first observed flame radius is assimilated into the model to obtain an estimated mass transfer number, $\hat{B}$. The corrected $\hat{B}$ is 0.580 using an analytical inverse and 0.355 with the numerical inverse for $n$-heptane. When predicting with the analytical model after assimilating data with the numerical model, the FSR is constant in time and less than the experimental value (Figure 3). The numerical predictions using parameters estimated with analytical inverse produce a significantly larger FSR than the experimental data. Moreover, these solutions do not reproduce the initial flame radius, indicating that the analytical solution is incompatible with the numerical solutions. This might be a consequence of the analytical solutions not quantifying mass transfer in the gas phase appropriately. The numerical predictions with numerical inverse, in contrast produce acceptable results. The estimated value of $\hat{B}$ for $\mathrm{n}$-heptane is 0.355 , and for $n$-decane the value is 0.295 . An important clarification is that only one observation is taken to calculate the value of $\hat{B}$, therefore, only one effective value of $\hat{B}$ is obtained and subsequently only one $C_{2}$ can be calculate. In this case $C_{2}$ is $4.15 \times 10^{-2}$ for $n$-heptane and is $3.52 \times 10^{-2}$ for n-decane and the fact that they are single numbers satisfy the needed condition of convergence for $C_{2}$. The RMSE between the predictions and observations is $8.46 \%$ for $n$-decane and $2.87 \%$ for $n$-heptane, respectively. It is clear that this effective mass transfer number incorporates other physical phenomena because the fact that it emerges as being less than unity appears to indicate that the heat generated and transferred from the flame to the surface is less than the energy necessary for gasification. In other words, the $\hat{B}$ 
is a parameter that "absorbs" all the errors generated from the imprecise input parameters, such as, initial-, boundary conditions and errors systemic to the model by changing $Q_{\text {loss }}$.

An evident difference between the assimilation method presented here and regression analysis is that in this method, the time-independent coefficients that represent concrete physical interpretations are corrected to steer the state of the system. For the statistical regression, the fitting curve only provides extrapolations to individual variables, thus predicting a future state of the system that consists of diverse variables is impossible. $\hat{K}$ and $\hat{B}$ therefore have to be time-independent.

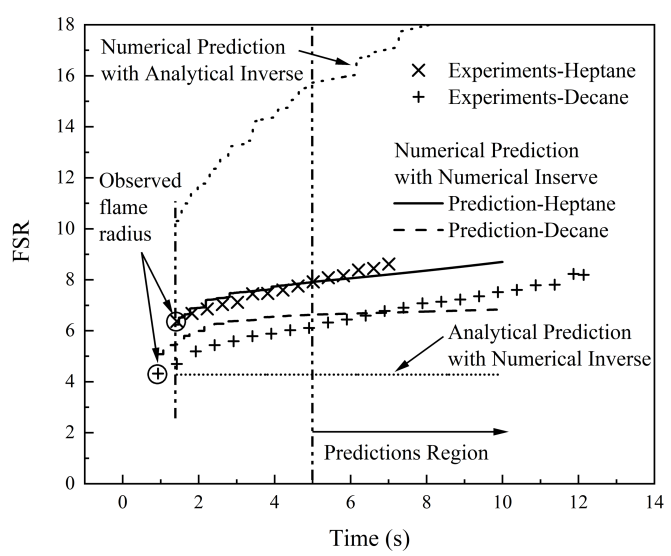

Figure 3: Different types of predictions for FSR with different types of inverse method for n-heptane droplet combustion. The best solutions correspond to numerical predictions with numerical inverse.

Figure 3 shows that the evolution of the flame stand-off distance diverges in time from the experimental values. The error is nevertheless acceptable, given the simplicity of the model and that a single observation is used to establish $\hat{B}$. Given that the droplet and flame diameters are the assimilated data, it is important to compare forecasts of other independent variables. The model provides also temperature and species mass fractions, and these are ideal candidates for comparison. However, the literature does not provide direct measurements of these parameters.

The peak gas temperature is estimated from another set of droplet combustion experiments with 
the support of a $80 \mu \mathrm{m}$ fiber rather than the free-floating droplets in FLEX [15]. The estimated gas temperature is calculated from the energy balance on the fiber with estimated heat transfer properties and the measured fiber temperature. By comparing the prediction for the assimilated free-floating droplet combustion with the fiber supported case, the performance of the assimilation can be evaluated even though they are from different experiments. The initial radius of the n-heptane droplet in the experiment is $1.6 \mathrm{~mm}$, which is similar to the free-floating case. Moreover, the authors report that the initial droplet size have little influence on the gas temperature.

Figure 4 shows the comparison between the computed and estimated peak temperatures. In general, the predictions and the experimental results have the same order of magnitude and tendency. The RMSE is $5.03 \%$. This indicates that the assimilated results from the free-floating droplet combustion have the potential to provide reasonable outputs for temperature. The use of infinite rate chemistry in the forward model leads to higher temperatures because the energy release is all concentrated in the flame sheet. The assimilated data used originates from a different experiment but the estimated peak temperature predicted remain reasonable showing which shows the adequacy of the methodology.

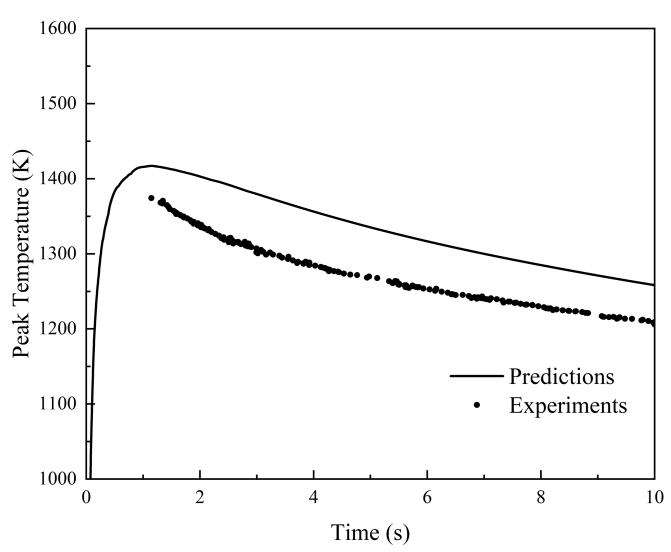

Figure 4: Peak temperature predictions for n-heptane droplet combustion 
In the absence of experimental data, detailed CFD simulations are used for comparison. For the species profiles, detailed simulation for n-decane combustion from a study carried out by Cuoci et al. [17] are used. In Cuoci's study, numerical calculations with 200 species, over 5000 reactions and detailed treatment towards the interface and droplet heating are conducted. The traditional droplet combustion model, which is the same as in the current work, with detailed chemistry kinetics is applied. It therefore corresponds to the the same conditions. The initial droplet size radius is $0.79 \mathrm{~mm}$, which is smaller than the assimilated case. However, Cuoci et al. showed that the initial size of the droplet will affect the combustion details only marginally. So, as with the temperature, the performance of the assimilation can be evaluated against the data presented by Cuoci et al. The species profiles from 1.2 $\mathrm{s}$ after the ignition, which is at the stable stage, are presented in Figure 5.
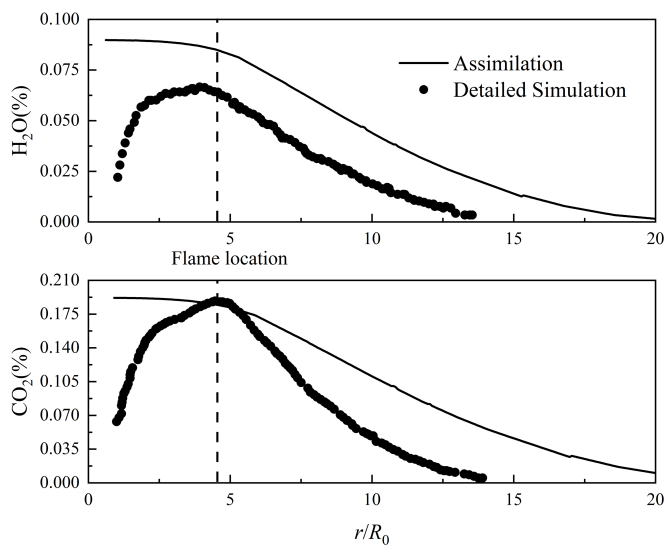

Figure 5: $\mathrm{H}_{2} \mathrm{O}$ and $\mathrm{CO}_{2}$ profile predictions n-decane droplet combustions at steady state

Figure 5 shows the species fractions for $\mathrm{CO}_{2}$ and $\mathrm{H}_{2} \mathrm{O}$ for the detailed simulation and the assimilated results. The profiles obtained from the simulations with the assimilated parameters are similar in shape to the detailed simulation and the flame location from assimilated results appears where the maximum occurs from the detailed simulation, but overpredict concentrations by up to $25 \%$. 
The RMSE is $61 \%$ for $\mathrm{H}_{2} \mathrm{O}$ profile and $53 \%$ for $\mathrm{CO}_{2}$ profile. The assimilated simulations cannot reproduce the species concentrations correctly between droplet surface and flame. The concentrations become constant close to the surface of the droplet because the product concentrations from the analytical solutions are injected to the numerical region as the boundary conditions. Still, even though different droplet sizes are considered and data from different experiments are assimilated, the prediction can compass enough physics and is robust enough reproduce the overall trends.

The proposed hybrid model exhibits important improvements in terms of run-time, when compared to the detailed simulations carried out by Cuoci et al.; the $10 \mathrm{~s}$ forecast including assimilation time took $32.35 \mathrm{~s}$ to execute on a i7-8650 $\mathrm{CPU}$ at $1.9 \mathrm{GHz}$. Cuoci's detailed simulation required 2 days on a Pentium IV at $2.8 \mathrm{GHz}$. It should be, in principle, possible to reduce the required resources further using local mesh refinement and coarser meshes away from the flaming region. If High Performance Computer (HPC) can be applied, the assimilation has great potentials to be utilized practically for forecasts with positive lead times.

\section{Extension for Droplet Combustion in Normal Gravity}

A useful extension of the proposed hybrid model would be to relax the micro-gravity assumption, thus predicting droplet combustion in normal gravity. It is to be expected that gravity affects the gas phase to a larger extent than the condensed phase, so that it is reasonable to maintain the spherical assumption for the analytical region, and simply to add a buoyancy term to the momentum equation in the numerical region in order to account for gravity. The location of the interface between the regions is maintained at the original droplet radius. For the interface, the heat flux is averaged using the same as method described above and the calculations for other boundary conditions remain the same.

The experimental data is for the n-heptane droplet combustion from Ambekar [18]. The droplet 
radius is measured by treating the droplet as a sphere, which is consistent for the proposed hybrid modelling approach. The elongation of the flame is driven by buoyancy, but is also associated with extinction chemistry [18], which makes the flame unstable. Thus, the irregularly shaped flame is here measured from the center of the droplet to the left boundary of the flame. The total observation time is $2 \mathrm{~s}$. The droplet size data from 0 to $1.2 \mathrm{~s}$ are used as observations for the estimation of $\hat{K}$ and the remaining data is compared to the predictions. The first data point for the flame size is used as observation to estimate $\hat{B}$.

Figure 6 shows the observations and predictions of the droplet combustion under normal gravity. The species will move upwards for buoyancy. The RMSE between the predictions and observations is $4.52 \%$. Although the droplet is treated as a sphere, assimilation still works satisfactorily, and the droplet size is steered towards the observed data. With the corrected $\hat{K}$, the predictions are thus acceptable.

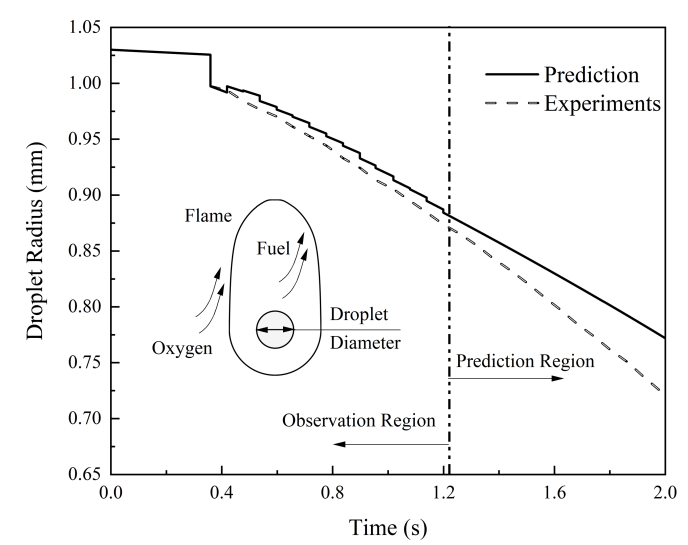

Figure 6: Droplet size observations and predictions for n-heptane droplet combustion with initial radius of $0.5 \mathrm{~mm}$ in normal gravity

Figure 7 shows the observations and predictions of the stable flame size (FSR). The RMSE in this case is $2.65 \%$. The simulations before $0.5 \mathrm{~s}$ fluctuate sharply because the model treats the interface 
as an injection surface. Thus the flame sheet will expand firstly. During the stable stage, the predictions of the flame size is closed to the posterior observations.

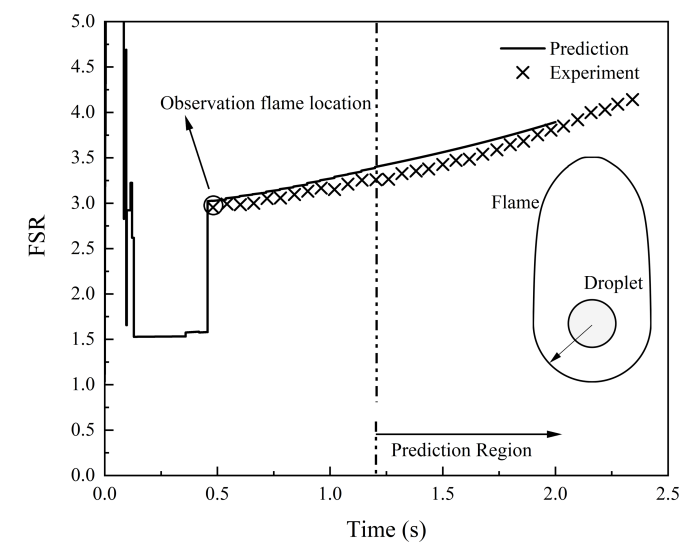

Figure 7: Flame shape observations and predictions for n-heptane droplet combustion in normal gravity

\section{Conclusions}

A novel modelling framework that consists of assimilating data into a combination of an analytical representation of gasification and a numerical representation for combustion is presented. The simplified representation of the interaction between the condensed and gas phases is an essential building block that will enable the construction of a modelling structure specially developed with data assimilation in mind and that could eventually be extended to more complex geometries. The framework delivers proper forecasts of droplet gasification rates, flame diameter, temperatures and species for droplets in microgravity. The approach also allows to incorporate buoyancy to predict the flame geometry and evaporation rates. These are all the key physical processes controlling a fire. Without the benefit of HPC or optimized numerical schemes, the computational times are sufficiently small to envision faster than real time forecasts. This study shows that extension of this approach to more complex geometries is viable and therefore it could be envisioned as a means to deliver fire 
predictions.

\section{References}

[1] E.N. Lorenz, The predictability of a flow which possesses many scales of motion, Tellus. 21 (3) (1969) 289-307.

[2] E. Kalnay, Atmospheric modeling, data assimilation and predictability, Cambridge University Press, Cambridge, UK, 2003.

[3] J. Janicka, A. Sadiki, Large eddy simulation of turbulent combustion systems, Proc. Combust. Inst. 30 (1) (2005) 537-547.

[4] B. Fiorina, D. Veynante, S. Candel, Modeling combustion chemistry in large eddy simulation of turbulent flames, Flow Turbul. Combust. 94 (1) (2015) 3-42.

[5] X. Gao, Y. Wang, N. Overton, M. Zupanski, X. Tu, Data-assimilated computational fluid dynamics modeling of convection-diffusion-reaction problems, J. Comput. Sci. 21 (2017) 38-59.

[6] H. Yu, T. Jaravel, M. Ihme, M. Juniper, L. Magri, Data Assimilation and Optimal Calibration in Nonlinear Models of Flame Dynamics, J. Eng. Gas Turbines Power. (2019).

[7] J.W. Labahn, H. Wu, B. Coriton, J.H. Frank, M. Ihme, Data assimilation using high-speed measurements and les to examine local extinction events in turbulent flames, Proc. Combust. Inst. 37 (2) (2019) 2259-2266.

[8] W. Jahn, G. Rein, J.L. Torero, Forecasting fire dynamics using inverse computational fluid dynamics and tangent linearisation, Adv. Eng. Softw. 47 (1) (2012) 114-126.

[9] W. Jahn, G. Rein, J. Torero, Forecasting fire growth using an inverse zone modelling approach, Fire Saf. J. 46 (2011) 81-88.

[10]W. Jahn, Using suppression and detection devices to steer CFD fire forecast simulations, Fire Saf. 
J. 91 (2017) 284-290.

[11]D.B. Spalding, The combustion of liquid fuels, Symp. Int. Combust. 4 (1) (1953) 847-864.

[12]G. Strotos, I. Malgarinos, N. Nikolopoulos, M. Gavaises, Predicting the evaporation rate of stationary droplets with the VOF methodology for a wide range of ambient temperature conditions, Int. J. Therm. Sci. 109 (2016) 253-262.

[13]D.L. Dietrich, V. Nayagam, M.C. Hicks, P.V. Ferkul, F.L. Dryer, T. Farouk, B.D. Shaw, H.K. Suh, M.Y. Choi, Y.C. Liu, C.T. Avedisian, F.A. Williams, Droplet Combustion Experiments Aboard the International Space Station, Microgravity Sci. Technol. 26 (2) (2014) 65-76.

[14]Y.C. Liu, Y. Xu, M.C. Hicks, C.T. Avedisian, Comprehensive study of initial diameter effects and other observations on convection-free droplet combustion in the standard atmosphere for nheptane, n-octane, and n-decane, Combust. Flame. 171 (2016) 27-41.

[15]D.L. Dietrich, R. Calabria, P. Massoli, V. Nayagam, F.A. Williams, Experimental Observations of the Low-Temperature Burning of Decane/Hexanol Droplets in Microgravity, Combust. Sci. Technol. 189 (3) (2017) 520-554.

[16]D.L. Dietrich, J.B. Haggard, F.L. Dryer, V. Nayagam, B.D. Shaw, F.A. Williams, Droplet combustion experiments in spacelab, Symp. Int. Combust. 26 (1) (1996) 1201-1207.

[17]A. Cuoci, M. Mehl, G. Buzzi-Ferraris, T. Faravelli, D. Manca, E. Ranzi, Autoignition and burning rates of fuel droplets under microgravity, Combust. Flame. 143 (3) (2005) 211-226.

[18]A. Ambekar, A. Chowdhury, An experimental technique for determination of intrinsic burning rate constants of liquid fuels, Appl. Therm. Eng. 135 (2018) 238-245.

\section{Supplement Material}

All the source codes and input files can be accessed on 
github.com/XiuqiXi/DatarhoReactingBuoyantFoam. 


\section{Figure captions}

Figure 1: The schematic of the forward model coupled with the analytical model and the numerical model

Figure 2: Droplet size observations and predictions for $10 \mathrm{~s}$ of n-heptane and n-decane droplet combustion. The right axis shows the value of $C_{1}=\hat{K} / K$.

Figure 3: Different types of predictions for FSR with different types of inverse method for n-heptane droplet combustion. The best solutions correspond to numerical predictions with numerical inverse.

Figure 4: Peak temperature predictions for n-heptane droplet combustion

Figure 5: $\mathrm{H}_{2} \mathrm{O}$ and $\mathrm{CO}_{2}$ profile predictions n-decane droplet combustions at steady state

Figure 6: Droplet size observations and predictions for n-heptane droplet combustion with initial radius of $0.5 \mathrm{~mm}$ in normal gravity

Figure 7: Flame shape observations and predictions for n-heptane droplet combustion in normal gravity 